\title{
Finite-Channel Chromatic Derivative Filter Banks
}

\author{
M. Cushman, Member, IEEE, M. J. Narasimha, Fellow, IEEE, and P. P. Vaidyanathan, Fellow, IEEE
}

\begin{abstract}
Two recent contributions discussed the theory of perfect-reconstruction (PR) chromatic derivative filter banks comprising an infinite number of channels. This letter extends the theory to the case of finite channels. A novel time domain procedure is delineated for designing the synthesis filters that achieve PR in this case.
\end{abstract}

Index Terms-Biorthogonal filter banks, chromatic derivatives, perfect reconstruction.

\section{INTRODUCTION}

$\mathbf{I}$ $\mathrm{N}$ THIS LETTER, we present the problem of multichannel sampling discussed in [3] and [4] from the view of a continuous-time biorthogonal filter bank, and we show that this viewpoint leads to new insights. A particular filter bank of this kind, called the chromatic derivative filter bank, is explored in greater detail. See [6] for a comprehensive survey of single and multichannel sampling theory.

Chromatic derivatives are linear combinations of the ordinary derivatives, where the coefficients of the combination are derived from orthogonal polynomial theory. Surprisingly, by taking combinations of a notoriously difficult operation such as differentiation, we obtain perfectly stable filters. This is because of the well-known orthogonal properties of the underlying polynomial set. The weighting function employed in the definition of these polynomials basically determines the characteristics of the filters. We believe that these derivatives are preferable to the ordinary derivatives in situations where wide-band channel splitting is needed. Multichannel analog-to-digital conversion of high-bandwidth signals is one such example.

The theory of perfect reconstruction (PR) in chromatic derivative filter banks with an infinite number of channels is discussed in [1] and [2]. We extend that theory to the finitechannel case here. Although one can obtain an approximate reconstruction of the original signal by truncating the infinite series expansions, as explained in [1], we show that it is in fact possible to achieve PR by proper choice of the synthesis filters. We delineate a time domain procedure for designing such filters.

Manuscript received May 28, 2002; revised August 1, 2002. The associate editor coordinating the review of this manuscript and approving it for publication was Dr. Markus Pueschel.

M. Cushman was with Kromos Technology, Los Altos, CA 94022 USA. He is now with Deephaven Capital Management, Santa Clara, CA 95054 USA (e-mail: mcushman@alumni.uchicago.edu).

M. J. Narasimha is with Stanford University, Stanford, CA 94305 USA (e-mail: sim@nova.stanford.edu).

P. P. Vaidyanathan is with the California Institute of Technology, Pasadena, CA 91125 USA (e-mail: ppvnath@systems.caltech.edu).

Digital Object Identifier 10.1109/LSP.2002.806706

\section{Biorthogonality in Finite-Channel ANalog FILTER BANKS}

Fig. 1 shows an $M$-channel filter bank, where the input signal is bandlimited to $|\omega|<\pi$, and the sampling rate of the subbands is $M$ times smaller than the Nyquist rate of one sample per second. The output signal can be expressed as $\hat{x}(t)=\sum_{k=0}^{M-1} \sum_{n=-\infty}^{\infty} v_{k}(n M) f_{k}(t-n M)$. We assume that the input $x(t)$ belongs to the space spanned by the doubly indexed set of (linearly independent) functions $f_{k}(t-n M), 0 \leq k \leq M-1,-\infty \leq n \leq \infty$, i.e., $x(t)=\sum_{k=0}^{M-1} \sum_{n=-\infty}^{\infty} a_{k, n} f_{k}(t-n M)$ for some set of constants $a_{k, n}$. For such an input, following the procedure in [1] and [2], we can show that the PR property holds if the analysis and synthesis filters satisfy the biorthogonality condition

$$
\begin{aligned}
\frac{1}{2 \pi} \int_{-\pi}^{\pi} H_{k}(\omega) F_{m}(\omega) e^{j \omega n M} d \omega= & \delta_{k m} \delta_{n 0}, \\
& 0 \leq k, m \leq M-1
\end{aligned}
$$

where $\delta_{k m}$ is the Kronecker delta function. If $g_{k m}(t)$ denotes the cascaded impulse response of the $k$ th analysis filter and the $m$ th synthesis filter, then (1) can equivalently be written as $g_{k m}(t)=$ $\delta_{k m} \delta_{n 0}$.

\section{Chromatic Derivatives Based on ORTHOGONAL POLYNOMIALS}

Consider a sequence of polynomials in the interval $[-1$, 1], which are orthogonal with respect to a nonnegative even weighting function that is not identically zero over the defining interval. It is well known [5] that such polynomials can be obtained using the recursion

$$
P_{n+1}(x)=b_{n} x P_{n}(x)-c_{n} P_{n-1}(x)
$$

with $P_{0}(x)=k_{0}$ and $P_{1}(x)=k_{1} x$. The values of the constants $k_{0}, k_{1}, b_{n}$, and $c_{n}$ depend on the weighting function that defines the polynomial family. As in [1], we identify the $k$ th analysis filter of the filter bank using the $k$ th orthogonal polynomial as follows:

$$
H_{k}(\omega)=j^{k} P_{k}(\omega / \pi), \quad 0 \leq k \leq M-1 .
$$

The factor $j^{k}$ in the definition of the analysis filters guarantees that their impulse responses $h_{k}(t)$ will be real for all values of $k$. The output $v_{k}(n M)$ of the $k$ th analysis filter in Fig. 1 is known as the $k$ th chromatic derivative of $x(t)$ evaluated at $t=n M$.

Using (2), it can be shown that the analysis filters are polynomials in $j \omega$. Specifically, we can express them as

$$
H_{k}(\omega)=\sum_{l=0}^{M-1} d_{k, l}(j \omega)^{l}, \quad 0 \leq k \leq M-1
$$




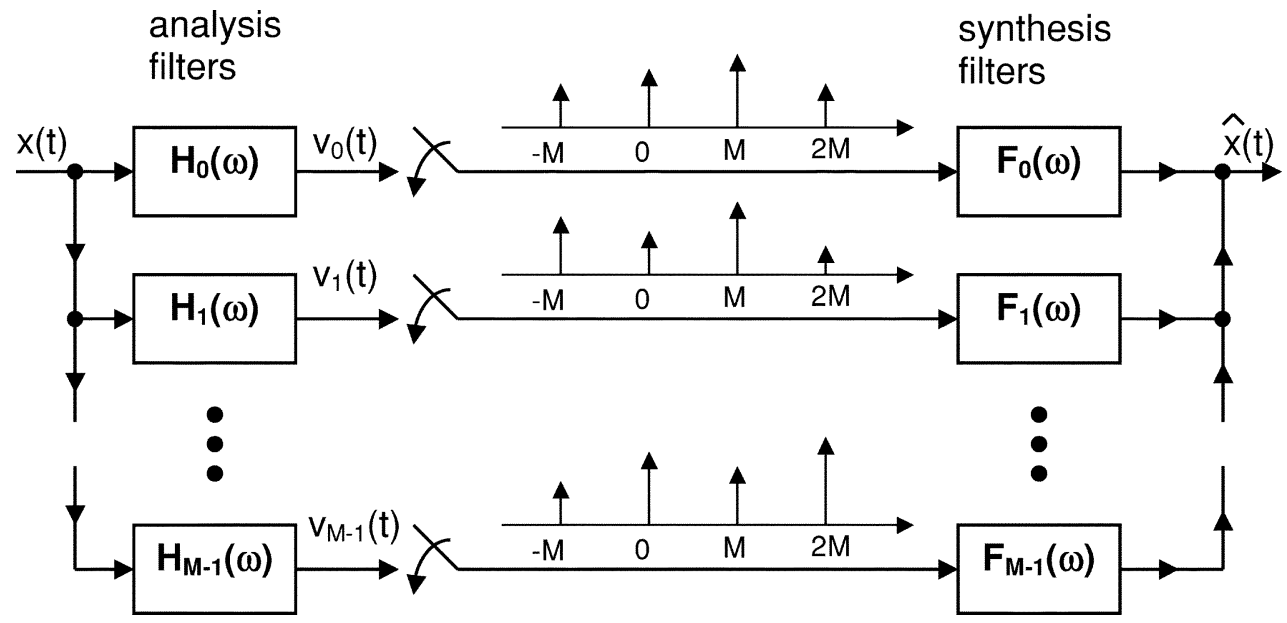

Fig. 1. $M$-channel filter bank for a signal $x(t)$ bandlimited to $|\omega|<\pi$.

where $d_{k, l}$ are real constants. If these constants are arranged as an $M \times M$ matrix $\mathbf{D}$ whose $k l$ th element is $d_{k, l}$, then it can be verified that the matrix is lower triangular with nonzero diagonal elements. This follows from the fact that the analysis filters satisfy a recursive relation similar to (2). Since the factor $(j \omega)^{l}$ in the frequency domain corresponds to the $l$ th derivative in the time domain, the matrix $\mathbf{D}$ also defines the relationship between the ordinary derivatives and the chromatic derivatives. (Note that the row and column indexing of the matrix starts at zero.)

\section{TIME DOMAIN Design OF SyNTHESIS FILTERS FOR PR}

With the analysis filters expressed as in (4), the biorthogonality or PR condition (1) becomes

$$
\begin{aligned}
\frac{1}{2 \pi} \int_{-\pi}^{\pi} \sum_{l=0}^{M-1} d_{k, l}(j \omega)^{l} F_{m}(\omega) e^{j \omega n M} d \omega & =\delta_{k m} \delta_{n 0}, \\
0 & \leq k, m \leq M-1 .
\end{aligned}
$$

Noting that $j \omega$ corresponds to differentiation in time, we can write this equivalently as

$$
\sum_{l=0}^{M-1} d_{k, l} f_{m}^{(l)}(n M)=\delta_{k m} \delta_{n 0}, \quad 0 \leq k, m \leq M-1
$$

for all $n$, with superscript $(l)$ denoting the $l$ th derivative. We now choose the synthesis filters as

$$
f_{m}(t)=p_{m}(t) s(t), \quad 0 \leq m \leq M-1
$$

where $s(t)$ is a $\pi$-bandlimited signal defined as the $M$ th power of the sinc function and $p_{m}(t)$ is a polynomial in $t$ of degree $M-1$

$$
s(t)=\left(\frac{\sin (\pi t / M)}{\pi t / M}\right)^{M}
$$

and

$$
p_{m}(t)=c_{0, m}+c_{1, m} t+\cdots+c_{M-1, m} t^{M-1} .
$$

The values $c_{q, m}, 0 \leq q \leq M-1$, represent the coefficients of the $m$ th polynomial. We will choose them so that the filter bank satisfies the PR conditions.

Using the Leibnitz rule for the $l$ th derivative of the product in (7), we get

$$
f_{m}^{(l)}(n M)=\sum_{q=0}^{l}\left(\begin{array}{l}
l \\
q
\end{array}\right) s^{(l-q)}(n M) p_{m}^{(q)}(n M) .
$$

Since $s(t)$ and its first $M-1$ derivatives are zero at $t=n M$ for all $n \neq 0$, it follows that $f_{m}(t)$ and its first $M-1$ derivatives are also zero at these points. Thus, the above choice of synthesis filters satisfies the PR condition (6) for all $n \neq 0$. Next, we pick the polynomial coefficients to take care of the case $n=0$.

Substituting (10) in (6) with $n=0$, and noting that $p_{m}^{(q)}(0)=$ $q ! c_{q, m}$, we get

$$
\sum_{l=0}^{M-1} d_{k, l} \sum_{q=0}^{l}\left(\begin{array}{l}
l \\
q
\end{array}\right) s^{(l-q)}(0) q ! c_{q, m}=\delta_{k m} .
$$

Using matrix notation, we can rewrite (11) as $\mathbf{D} \mathbf{\Delta} \mathbf{C}=\mathbf{I}$, where $\mathbf{D}$ is the $M \times M$ matrix that relates the ordinary derivatives to the chromatic derivatives as explained in Section III; $\mathbf{C}$ is an $M \times M$ matrix of the polynomial coefficients with $q m$ th element $c_{q, m}$; and $\boldsymbol{\Delta}$ is an $M \times M$ lower triangular matrix with elements

$$
[\boldsymbol{\Delta}]_{\mathbf{l}, \mathbf{q}}= \begin{cases}\left(\begin{array}{l}
l \\
q
\end{array}\right) s^{(l-q)}(0) q !, & q \leq l \\
0, & q>l .\end{cases}
$$

The diagonal elements of $\boldsymbol{\Delta}$ are nonzero, ensuring nonsingularity. The synthesis filters can, therefore, be uniquely identified from the elements of the matrix $C=\Delta^{-1} \mathbf{D}^{-1}$. Since $\Delta$ and $\mathbf{D}$ are both lower triangular, $\mathbf{C}$ is also a lower triangular matrix. Its $m$ th column denotes the coefficients of the $m$ th polynomial. Specifically, we can write $p_{m}(t)=c_{m, m} t^{m}+c_{m+1, m} t^{m+1}+$ $\cdots+c_{M-1, m} t^{M-1}$. 


\section{Example}

Let $M=4$, and assume that the analysis filters are based on the Chebyshev family of chromatic derivatives [1]. In this case, the matrices are given by

$$
\mathbf{D}=\left(\begin{array}{cccc}
1 & 0 & 0 & 0 \\
0 & 1 / \pi & 0 & 0 \\
1 & 0 & 2 / \pi^{2} & 0 \\
0 & 3 / \pi & 0 & 4 / \pi^{3}
\end{array}\right)
$$

and

$$
\boldsymbol{\Delta}=\left(\begin{array}{cccc}
1 & 0 & 0 & 0 \\
0 & 1 & 0 & 0 \\
-\pi^{2} / 12 & 0 & 2 & 0 \\
0 & -\pi^{2} / 4 & 0 & 6
\end{array}\right)
$$

The corresponding polynomials are $p_{0}(t)=1-\left(5 \pi^{2} / 24\right) t^{2}$, $p_{1}(t)=\pi t-\left(\pi^{3} / 12\right) t^{3}, p_{2}(t)=\left(\pi^{2} / 4\right) t^{2}$, and $p_{3}(t)=$ $\left(\pi^{3} / 24\right) t^{3}$.

\section{CONCLUSION}

The interpretation of generalized sampling as a continuous-time biorthogonal filter bank leads to many novel sampling expansions. One such expansion based on chromatic derivatives is discussed in this letter, along with an elegant time domain design procedure for the synthesis filters to achieve PR. Our procedure is simpler than the frequency domain approach proposed in [3] for the case of ordinary derivative-based sampling. The realization of a stable filter bank in which the channel filters are all wideband is possible by employing chromatic derivative operators for the analysis filters. Potential applications of this filter bank include multichannel analog-to-digital conversion, where sub-Nyquist samples of the individual channels can be used to represent a broadband signal, and transient analysis of signals.

\section{REFERENCES}

[1] M. J. Narasimha, A. Ignjatovic, and P. P. Vaidyanathan, "Chromatic derivative filter banks," IEEE Signal Processing Lett., vol. 9, pp. 215-216, July 2002.

[2] P. P. Vaidyanathan, A. Ignjatovic, and M. J. Narasimha, "New sampling expansions for bandlimited signals based on chromatic derivatives," in Proc. 35th Asilomar Conf. Signals, Systems, and Computers, Monterey, CA, Nov. 2001.

[3] A. Papoulis, "Generalized sampling expansion," IEEE Trans. Circuits Syst., vol. CAS-24, no. 11, pp. 652-654, Nov. 1977.

[4] J. L. Brown, Jr., "Multi-channel sampling of lowpass signals," IEEE Trans. Circuits Syst., vol. CAS-28, pp. 101-106, Feb. 1981.

[5] G. Szegö, Orthogonal Polynomials, 4th ed, ser. AMS Colloquium Publications. Providence, RI: Amer. Math. Soc., 1981, vol. 23, ch. II.

[6] M. Unser, "Sampling-50 years after Shannon," Proc. IEEE, vol. 88, pp. 569-587, Apr. 2000. 